\title{
Alternative distributions for the multiplicative model in SAR images
}

\author{
Alejandro C. Frery \\ Corina da Costa Freitas Yanasse \\ Sidnei J. S. Sant'Anna \\ INPE - Instituto Nacional de Pesquisas Espaciais \\ DPI - Divisão de Processamento de Imagens \\ Avenida dos Astronautas, 1758 \\ 12227-010 São José dos Campos \\ SP - BRAZIL \\ T: +55 123418977 Ext. 533 F: +55 123218743 EMail: [frery; corina; sidnei] edpi.inpe.br
}

\begin{abstract}
Another class of distributions is proposed to model the terrain backscatter, namely the class of the generalized inverse Gaussian distributions. Besides allowing the explicit calculation of the density of the random variable that models the radar return, these distributions have the remarkable property of having the gamma and other distributions as particular cases. The resulting distributions for complex, multilook intensity and multilook amplitude data are derived assuming the multiplicative model. These new densities yield to a more general model than the classical one, which is given by the class of $K$ distributions. Several plots are presented, showing the flexibility of these new distributions and its possible use as a model for SAR data.
\end{abstract}

\section{INTRODUCTION AND SAR IMAGE FORMATION}

The multiplicative model has been widely used in the modelling, processing and analysis of synthetic aperture radar images. It assumes that the observations within this kind of image are the outcome of a random variable that can be decomposed in the product of two other independent random variables: one $(Y)$ modelling the speckle noise, and the other $(X)$ modelling the terrain backscatter - which is always considered real positive-. Therefore, the observed value is the outcome of the random variable defined by the product $Z=X Y$. Three types of data are considered in this work, namely complex, intensity, and amplitude; their representation with the multiplicative model will be denoted using the indexes " $C$ ", " $~ I$ ", and " $A$ " respectively.

For complex data it is usually assumed that the speckle noise is a pair (vector) of independent identically distributed Gaussian random variables $Y_{C}=\left(Y_{\mathbf{s}}, Y_{\mathbf{s}}\right)$ with zero mean and variance $\sigma^{2}=1 / 2$ (this distribution is denoted by $\mathcal{N} 2(0,1 / 2)$ ), and complex data is modelled as $Z_{C}=\left(Z_{s}, Z_{\S}\right)=X_{A} Y_{C}$. One look intensity data is formed taking the modulus of $Z_{c}$, namely $Z_{l}=X_{A}^{2} \cdot\left|Y_{d}\right|^{2}$; while $n$-looks data is obtained averaging one look independent intensity observations, i.e. $Z_{I}=X_{A}^{2} n^{-1} \sum_{i=\mathbb{i}}^{n}\left|Y_{C}(i)\right|^{2}$. Amplitude data is obtained taking the square root of intensity data. These assumptions are well justified by the physics of illumination, which uses coherent radiation to form the image.

Using the aforementioned hypothesis $Y_{I}$ has a gamma distribution, denoted here $Y_{I} \sim \Gamma(\alpha, \lambda)$, characterized by the density

$$
f_{Y_{I}}(x ; \alpha, \lambda)=\lambda^{\alpha} /(\Gamma(\alpha)) x^{\alpha-1} \exp (-\lambda x), \quad x, \alpha, \lambda>0,
$$
with $\alpha=\lambda=n$. The amplitude speckle is characterized by the square root of gamma distribution, denoted $\sqrt{\Gamma}(\alpha, \lambda)$, whose density is

$$
f_{Y_{A}}(x ; \alpha, \lambda)=2 \lambda^{\alpha} /(\Gamma(\alpha)) x^{2 \alpha-1} \exp \left(-\lambda x^{2}\right), x, \alpha, \lambda>0,
$$
with $\alpha=\lambda=n$.
In order to model the terrain backscatter $\left(X_{A}\right)$, several distributions could be used. In particular, the distribution of a constant (a probability mass equal to one) seems to be well suited to homogeneous areas, whilst a square root of gamma distribution has been used for inhomogeneous targets. The former leads to model $Z_{C}\left(Z_{A}, Z_{I}\right)$ as a normal distribution (square root of gamma and gamma distributions, resp.), while the latter leads to $K$ distributions $[2,7]$.

$K$ distributions are characterized by the following densities: $f_{z_{\mathbf{x}}}(x ; \alpha, \lambda)=f_{z_{\boldsymbol{g}}}(x ; \alpha, \lambda)=\frac{2 \lambda^{(\alpha+1 / 2) / 2}}{\sqrt{\pi} \Gamma(\alpha)}|x|^{\alpha-1 / 2} K_{a-1 / 2}(2|x| \sqrt{\lambda})$, $f_{z_{l}}(x, \alpha, \beta, n)=\frac{2 \alpha n}{\beta \Gamma(\alpha) \Gamma(n)}\left(\frac{\alpha n x}{\beta}\right)^{(\alpha+n) / 2-1} K_{\alpha-n}(2 \sqrt{\alpha n x / \beta})$, $f_{Z_{A}}(x ; \alpha, \beta, n)=\frac{4 \alpha n x}{\beta \Gamma(\alpha) \Gamma(n)}\left(\frac{\alpha n x^{2}}{\beta}\right)^{(\alpha+n) / 2-1} K_{\alpha-n}(2 x \sqrt{\alpha n / \beta})$, where $K_{\mathrm{v}}$ is the modified Bessel function of the third kind and order $v, \alpha, \lambda>0, \alpha / \lambda=\beta$ and $x \in R$ for the first and $x \in \mathbf{R}_{+}$for the last two densities. These distributions are denoted by, respectively, $\operatorname{GCC}(\alpha, \lambda), \operatorname{gGI}(\alpha, \beta, n)$ and $\operatorname{I6A}(\alpha, \beta, n)$. The $r$-th order moments of these distributions are

$$
\begin{aligned}
& \mathrm{E}\left(Z_{\aleph}\right)= \begin{cases}0 & \text { if } r \text { is odd } \\
\frac{\Gamma(\alpha+r / 2)}{(2 \lambda)^{r / 2} \Gamma(\alpha)}(1 \cdot 3 \cdot \ldots \cdot(r-1)) & \text { if } r \text { is even }\end{cases} \\
& \mathrm{E}\left(Z_{l}\right)=\frac{K_{a+r}(2 \sqrt{\lambda \gamma}) \Gamma(n+r)}{K_{a}(2 \sqrt{\lambda \gamma}) \Gamma(n)}\left(\frac{\gamma}{n^{2} \lambda}\right)^{r / 2} \\
& \mathrm{E}\left(Z_{A}^{r}\right)=\mathrm{E}\left(Z_{I}^{r / 2}\right) .
\end{aligned}
$$

The assumption of a square root of gamma distribution for the modelling of the amplitude backscatter (or, equivalently, a gamma distribution for the intensity backscatter) is mainly based on empirical evidence, though some theoretical results can be found in [2]. It is a very convenient hypothesis, since it allows the explicit derivation of the resulting density. Many other distributions for the backscatter do not have this property as, for instance, it is not possible to obtain -in general - the density of the random variable defined as the product of a gamma distributed random variable by a normally distributed one.

In this paper, the use of the generalized inverse Gaussian distribution is proposed as a more general model for the intensity backscatter, and the related densities are derived. This distribution is characterized by the density

$$
f_{X_{I}}(x ; \alpha, \gamma, \lambda)=\frac{(\lambda / \gamma)^{\alpha / 2}}{2 K_{\alpha}(2 \sqrt{\lambda \gamma})} x^{\alpha-1} \exp \left(-\frac{\gamma}{x}-\lambda x\right), x>0 .
$$

It is denoted $X_{l} \sim \mathcal{N}^{-1}(\alpha, \gamma, \lambda)$, with parameters domain given by 


$$
\begin{cases}\gamma>0, \lambda \geq 0 & \text { if } \alpha<0, \\ \gamma>0, \lambda>0 & \text { if } \alpha=0, \\ \gamma \geq 0, \lambda>0 & \text { if } \alpha>0 .\end{cases}
$$

The $r$-th order moments of a $\mathcal{N}^{-1}(\alpha, \gamma, \lambda)$ distributed random variable are given by

$$
\mathrm{E}\left(X_{l}^{r}\right)=\frac{K_{a+r}(2 \sqrt{\gamma \lambda})}{K_{\mathrm{a}}(2 \sqrt{\gamma \lambda})}\left(\frac{\gamma}{\lambda}\right)^{r / 2}
$$

It can be proved that this distribution has the following important particular cases:

- the gamma distribution, since $X_{I} \sim \Gamma(\alpha, \lambda)$ if $\gamma=0$;

- the distribution of the reciprocal of a gamma distributed

random variable, if $\lambda=0$;

- the inverse Gaussian distribution, if $\alpha=-1 / 2$;

- the distribution of a reciprocal inverse Gaussian variate, if

$\alpha=1 / 2$, and

- the hyperbola distribution, when $\alpha=0$.

This distribution was proposed in [3], and it has found many remarkable applications $[1,5,6]$.

The distribution of the square root of a $\mathcal{N}^{-1}(\alpha, \gamma, \lambda)$ distributed random variable will be used to model the amplitude backscatter. Its density, denoted by $\mathcal{N}^{-1 / 2}(\alpha, \gamma, \lambda)$, is given by

$$
f_{X_{\Lambda}}(x ; \alpha, \gamma, \lambda)=\frac{(\lambda / \gamma)^{\alpha / 2}}{K_{\alpha}(2 \sqrt{\lambda \gamma})} x^{2 \alpha-1} \exp \left(-\frac{\gamma}{x^{2}}-\lambda x^{2}\right), x>0,
$$

and its $r$-th order moments are given by $\mathrm{E}\left(X_{\Lambda}^{r}\right)=\mathrm{E}\left(X_{I}^{r / 2}\right)$, whith $\mathrm{E}\left(X_{I}\right)$ given in eq. (2).

\section{THE G DISTRIBUTIONS}

In [4] an extension of $K$ distributions is presented, using a drift in the random walk that generates the speckle noise. In this paper these distributions are extended to a different distribution using the square root of generalized inverse Gaussian distribution as the model for the amplitude backscatter, while keeping the complex speckle noise as a pair of independent identically distributed Gaussian random variables.

Using the multiplicative model $Z_{C}=X_{A} Y_{C}$, and assuming that $X_{A} \sim \mathcal{N}^{-1 / 2}(\alpha, \gamma, \lambda)$ and $Y_{C} \sim \mathcal{N} 2(0,1 / 2)$ are independent, then it can be proved that the density of either component of $Z_{C}$ is given by

$$
\begin{array}{r}
f_{z_{3}}(z ; \alpha, \gamma, \lambda)=f_{z_{\Im}}(z ; \alpha, \gamma, \lambda)=\frac{(\lambda / \gamma)^{\alpha / 2}}{\sqrt{\pi} K_{\alpha}(2 \sqrt{\lambda \gamma})} . \\
\left(\left(\gamma+z^{2}\right) / \lambda\right)^{(\alpha-1 / 2) / 2} K_{\alpha-1 / 2}\left(2 \sqrt{\lambda\left(\gamma+z^{2}\right)}\right) .
\end{array}
$$

This distribution, denoted here as $\mathcal{G} C(\alpha, \gamma, \lambda)$, has been studied in [1] where it is a special case of the generalized hyperbolic distributions, and is denoted by $\mathcal{H}(\alpha, 2 \sqrt{\lambda}, 0, \sqrt{\gamma}, 0)$. Its parameters domain is given in eq. (1). It is symmetric and unimodal, with the mode at the origin; its $r$-th order moments are given by

$$
\begin{aligned}
& \mathrm{E}\left(Z_{s}^{r}\right)=\mathrm{E}\left(Z_{\mathrm{g}}^{r}\right)= \\
& =\left\{\begin{array}{l}
0 \quad \text { if } r \text { is odd } \\
\frac{K_{a+r / 2}(2 \sqrt{\gamma \lambda})}{K_{\alpha}(2 \sqrt{\gamma \lambda})}\left(\frac{\gamma}{4 \lambda}\right)^{r / 4}(1 \cdot 3 \cdot \ldots \cdot(r-1)) \text { if } r \text { is even }
\end{array}\right.
\end{aligned}
$$

Now turning to the intensity case, it is possible to prove that the distribution of the product of two indenpendent random variables $X_{I} \sim \mathcal{N}^{-1}(\alpha, \gamma, \lambda)$ and $Y_{I} \sim \Gamma(n, n)$ is characterized by the density

$$
\begin{array}{r}
f_{z_{l}}(x ; \alpha, \gamma, \lambda, n)=\frac{n^{n}(\lambda / \gamma)^{\alpha / 2}}{\Gamma(n) K_{\alpha}(2 \sqrt{\lambda \gamma})} x^{n-1}\left(\frac{\gamma+n x}{\lambda}\right)^{(\alpha-n) / 2} \\
K_{\alpha-n}(2 \sqrt{\lambda(\gamma+n x)}), \quad x>0
\end{array}
$$

with $n>0$ and the remaining parameters domain given in eq. (1). This distribution is denoted $Z_{I}-\mathcal{G} I(\alpha, \gamma, \lambda, n)$; its $r$-th order moments are given by

$$
\mathrm{E}\left(Z_{I}^{\prime}\right)=\frac{K_{\mathrm{a}+r}(2 \sqrt{\gamma \lambda}) \Gamma(n+r)}{K_{\alpha}(2 \sqrt{\gamma \lambda}) \Gamma(n)}\left(\frac{1}{n} \sqrt{\frac{\gamma}{\lambda}}\right)^{r} .
$$

It is possible to see that this distribution includes the aforementioned $96 I$ distribution, since it can be proved that $\operatorname{Gr}(\alpha, 0, \lambda, n)=\operatorname{Gr}(\alpha, \alpha / \lambda, n)$, where the sign " $="$ should be understood as "equivalence between distributions".

If amplitude, rather than intensity, data are considered, the distribution of the echo return is the distribution of the product of two independent random variables $X_{A}-\mathcal{N}^{-1 / 2}(\alpha, \gamma, \lambda)$ and $Y_{A} \sim \sqrt{\Gamma}(n, n)$, which is given by the density

$$
\begin{array}{r}
f_{Z_{A}}(x ; \alpha, \gamma, \lambda, n)=\frac{2 n^{n}(\lambda / \gamma)^{\alpha / 2}}{\Gamma(n) K_{\alpha}(2 \sqrt{\lambda \gamma})} x^{2 n-1}\left(\frac{\gamma+n x^{2}}{\lambda}\right)^{(\alpha-n) / 2} . \\
K_{\alpha-n}\left(2 \sqrt{\lambda\left(\gamma+n x^{2}\right)}\right), x>0 .
\end{array}
$$

Its parameters domain is the same of eq. (3). This distribution is denoted $Z_{A}-G A(\alpha, \gamma, \lambda, n)$; its $r$-th order moments are given by

$$
\mathrm{E}\left(Z_{A}\right)=\frac{K_{\alpha+r / 2}(2 \sqrt{\gamma \lambda}) \Gamma(n+r / 2)}{K_{\alpha}(2 \sqrt{\gamma \lambda}) \Gamma(n)}\left(\frac{\gamma}{n^{2} \lambda}\right)^{r / 4} .
$$

It is possible to prove that, setting $\gamma=0$ and $\beta=\alpha / \lambda$, the QA distribution becomes the distribution of the square root of a $96 J(\alpha, \beta, n)$ distributed random variable.

Figs. 1 to 4 show examples of the distributions presented in this work for three different types of areas: homogeneous $(H)$, moderately heterogeneous (M) and extremely heterogeneous (E). Fig. 1 shows the densities associated to the backscatters of $\mathrm{H}$ (a constant equal to $\sqrt{5000}), \mathrm{M}(\sqrt{\Gamma}(2,2 / 5000))$ and $E\left(\mathcal{N}^{-1 / 2}\left(-3,10^{4}, 2.96 \cdot 10^{-15}\right)\right)$. These parameters were chosen to generate complex distributions with the same first and second moments.

In Fig. 2 the resulting complex distributions are shown: $H$ (a Gaussian distribution with zero mean and variance 2500$), \mathrm{M}$ $(96 C(2,2 / 5000))$ and $\mathrm{E}\left(\mathrm{GC}\left(-3,10^{4}, 2.96 \cdot 10^{-15}\right)\right)$. Fig. 3 shows the associated intensity densities to $H(\Gamma(1,1 / 5000))$, $\mathrm{M}(96 \mathrm{~J}(2,5000,1))$ and $\mathrm{E}\left(\mathrm{GI}\left(-3,10^{4}, 2.96 \cdot 10^{-15}, 1\right)\right)$. Fig. 4 presents the associated amplitude densities to the three types of areas: $H(\sqrt{\Gamma}(1,1 / 5000)), M(96 A(2,5000,1))$ and $E$ $\left(G A\left(-3,10^{4}, 2.96 \cdot 10^{-15}, 1\right)\right)$.

It is interesting to note that, though all the complex distributions have zero means and variances equal to 2500 , the associated GI variance in the intensity domain is larger than the corresponding $96 I$ variance which, in turn, is larger than the $\Gamma$ one, though they all have the same intensity mean. One implication of this fact is that, for the same intensity mean, the inclusion of new parameters yields to distributions that allow larger variances and, with higher probabilities, the appearance 
of larger backscatter values; in this way, heterogeneous areas can be modelled better.

\section{CONCLUSIONS AND FUTURE WORK}

It has been shown that the use of $\boldsymbol{N}^{-1}$ distributions for the intensity backscatter yields to a more general model for SAR data than the use of $\Gamma$ distributions, whilst preserving (and extending) all the relations presented in [7]. The same idea could be applied to the extension of the speckle.

Parameters estimation procedures are being studied for the $G C, g I$ and $G A$ distributions. The discriminatory capabilities of its estimated parameters are also under evaluation.

\section{ACKNOWLEDGMENTS}

The authors are grateful to Prof. Ole E. Barndorff-Nielsen (Institute of Mathematics, University of Ärhus, Denmark) for his suggesting the use of inverse Gaussian distributions and for his warm reception of one of the authors (ACF) of this work. This work was partially supported by FAPESP (Fundação de Amparo à Pesquisa do Estado de São Paulo, Brazil) Project No. 91/3532-2, by CNPq and by ABC (the Brazilian Academy of Sciencies).

\section{REFERENCES}

[1] O.E. Barndorff-Nielsen and P. Blæsild, "Hyperbolic distributions and ramifications: contributions to theory

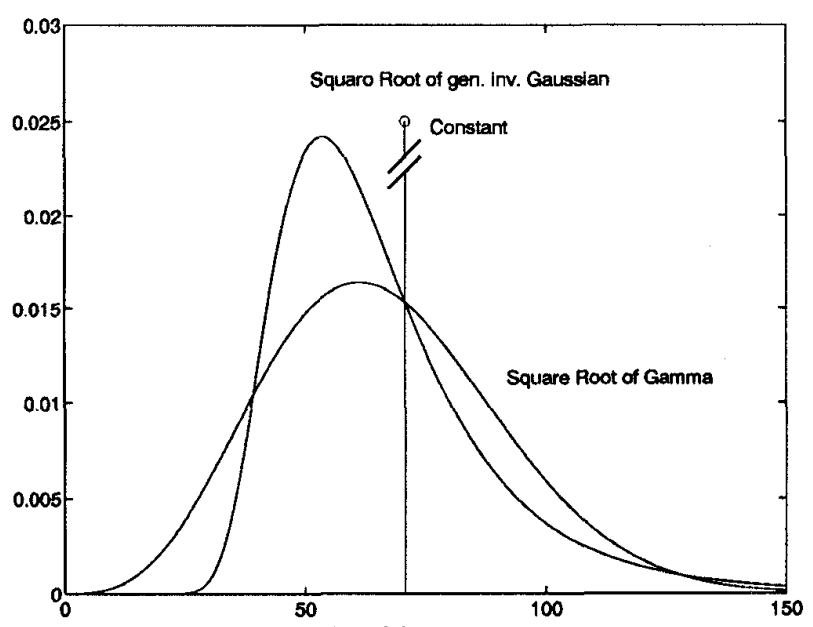

Fig. 1: Backscatter densities.

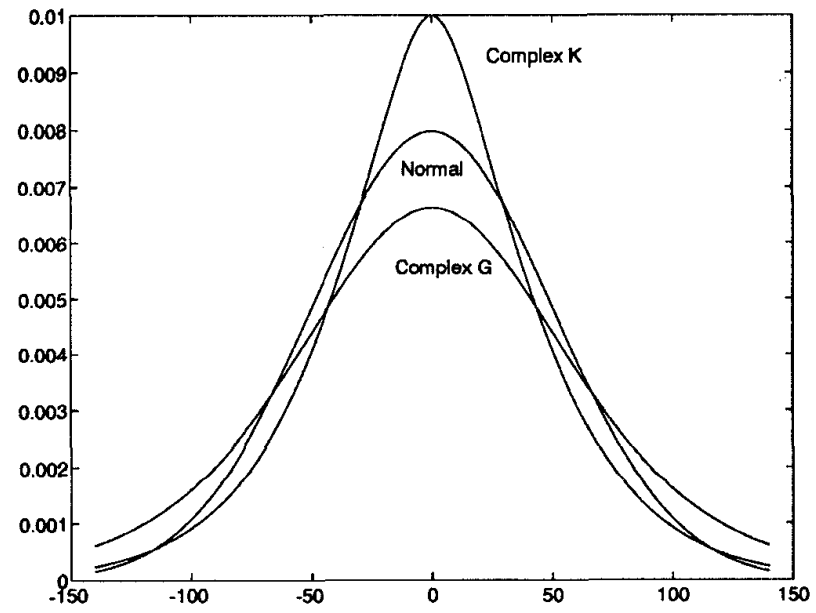

Fig. 2: Complex return densities. and applications," in: Statistical Distributions in Scientific Work, vol. IV. C. Taillie, G.P. Patil and B.A. Baldessari, Eds. Dordrecht, Reidel, 1981, pp. 19-44.

[2] R.G. Caves, Automatic matching of features in synthetic aperture radar data to digital map data. Sheffield, UK: University of Sheffield, 1993, $228 \mathrm{pp}$. (PhD Thesis).

[3] I.J. Good, "The population frequencies of species and the estimation of population parameters," Biometrika, vol. 40, pp. 237-260, 1953.

[4] E. Jakeman and R.J.A Tough, "Generalized $K$ distribution: a statistical model for weak scattering," J. Opt. Soc. Am. A, vol. 4, pp. 1764-1772, 1987.

[5] B. Jørgensen, Statistical properties of the generalized inverse Gaussian distribution. New York, Springer-Verlag, 1982. (Lecture Notes in Statistics, 9).

[6] H.S. Sichel, "On a distribution representing sentence-length in written prose," J.R. Statist. Soc. A, vol. 137, pp. 25-34, 1974.

[7] C.C.F. Yanasse, A.C. Frery and S.J.S. Sant'Anna, "Stochastic distributions and the multiplicative model: relations, properties, estimators and applications to SAR image analysis," unpublished.

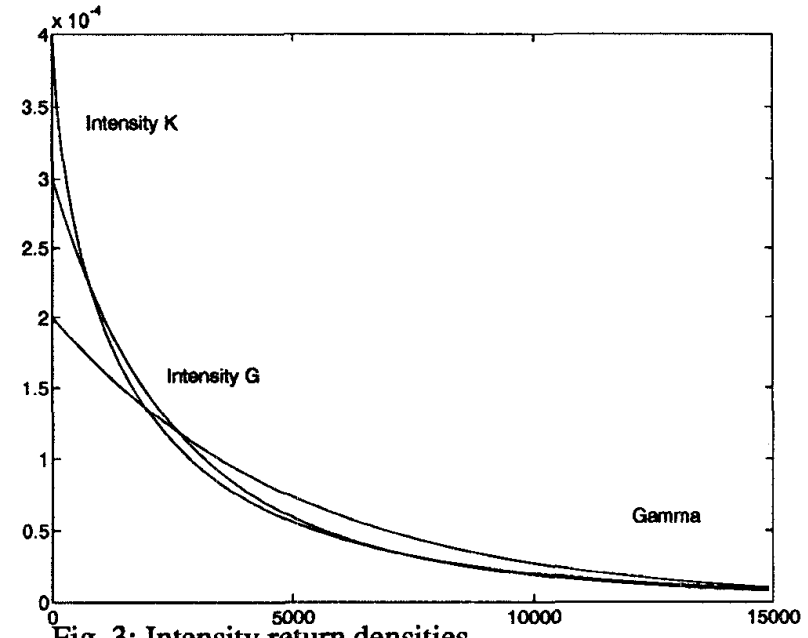

Fig. 3: Intensity return densities.

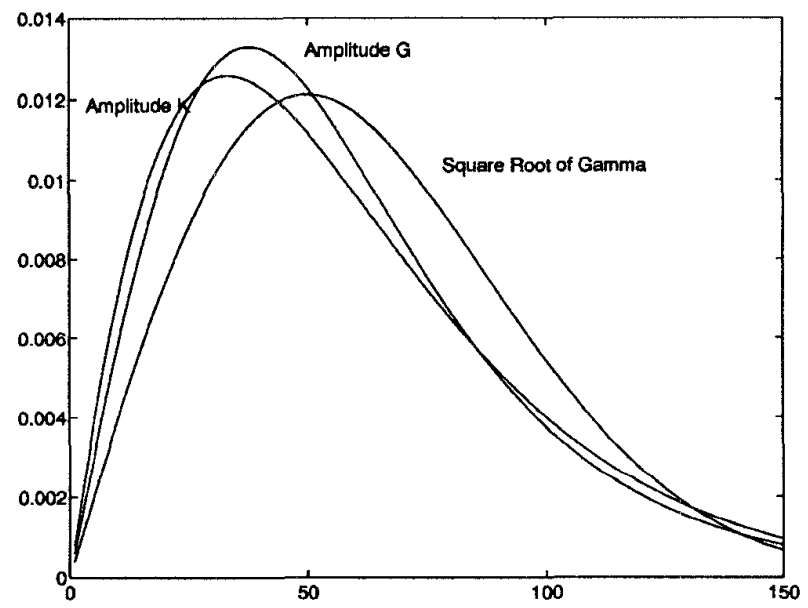

Fig. 4: Amplitude return densities. 